\title{
JEALOUSLY IN THE LAB: THE EFFECT OF A THIRD PARTY INVESTMENT IN THE ROMANTIC PARTNER
}

\author{
María Teresa Barbato1, Ana María Fernández² and Carlos Rodriguez-Sickert1 \\ ${ }^{1}$ Social Complexity Research Center (CICS), Universidad del Desarrollo, Santiago, Chile \\ ${ }^{2}$ Laboratory of Evolution and Interpersonal Relationships (LERI), Universidad de Santiago, \\ Santiago, Chile
}

mtbarbat@uc.cl

\begin{abstract}
In long-term mating, individuals take advantage of all the benefits inherent to a cooperative heterosexual relationship. If we consider that natural selection produced sex differences in the design of adaptations to solve the problems surrounding reproduction, then the design of human jealousy, which is an emotion triggered by lost of a valued relationship, must also be triggered by distinct evoking acts that are specific adaptive challenges for women and men in their exclusivity of their pair-bond. We present a pilot study with a novel method to experimentally trigger the adaptive sex-differences in jealousy. Specifically, we use a game theory protocol in which each member of 28 committed couples $(n=56)$ participated in two interpersonal dictator games against an opposite sex third party and a control condition. In the first dictator game, each member of the dyad performs the role of allocator. In the second game, the members of the couple perform the role of the recipient. The outcome of both games is informed to the partner (jealousy evoking protocol). We hypothesize that i) self-reported evoked jealousy will be greater for women when informed about the outcome of the game in which her partner plays the role of the allocator (the game represents a situation in which their male partner invests resources in another female); and conversely, ii) self-reported jealousy will be greater for male subjects when their partner plays the role of the recipient (the game represents a situation in which his female partner receives resources from another male). The results show that this protocol exerted the expected evocation of jealousy for both sexes. We discuss sex-differences in the treatments and possible alternative modifications to improve the similarity of the game to actual jealousy.
\end{abstract}

Keywords: Mating, Human Mating, Emotions. 


\section{INTRODUCTION}

An open question in human mating is whether jealousy is caused by general distrust on the exclusivity of partner's affection (Chin et al., 2017; Ellis \& Kleinplatz, 2018), or whether it is an evolved reaction caused by an interloper that can potentially attract away the partner and replace us (Buss, Larsen, Westen, \& Semmelroth, 1992; Buunk, 1997; Dillon, 2013). Natural selection led to sex differences in the design of psychological adaptations for solving the problems surrounding reproduction, triggered by distinct ancestral problems which become specific challenges for women and men in the exclusivity of their reproductive bond (Buss, 2013, 2018). From an evolutionary perspective, jealousy would be directed towards driving away interlopers who could potentially threaten the bond with the reproductive partner (Buss, 2018; Dijkstra and Buunk, 2002).

Reproductive relationships are dynamic processes based on social exchange (Conroy-Beam, Goetz, \& Buss; 2015; Cosmides \& Tooby, 2013), and in order to end-up in reproduction there are two evolutionary challenges to this kind of bonding: mate choice and mate retention (Buss, 1989, 2018). Specifically, mate retention evolved since losing a partner is more expensive than facing the costs of suspecting and/or acting on a false probability of being cheated (Buss and Haselton, 2005a; Foster et al., 2014 Schmitt \& Buss, 2001). Thus, natural selection shaped cognitive bias to deter the possible betrayal from a partner and increasing protection of the mating bond. Therefore, jealousy can be considered an emotion alerting the individual to engage in and executing the most effective mechanisms to prevent cheating by a partner, driving away mating rivals (Buss, 2018; Buss and Haselton, 2005b).

\section{Origins of Jealousy}

Jealousy has been proposed as an adaptive emotion that develops across ontogeny, when close attachment, or romantic bonding is disrupted (Buss, 2013; Dillon, 2013; Hart, 2017). Others have suggested that jealousy may be a maladaptive emotion emerging from exacerbated personal insecurity (Chin et al., 2017). But contrary to this, evolutionary thought (Buss, 2013, 2018) has led to the notion that sexual jealousy is an evolved emotion directed at driving away individuals perceived as rivals, which potentially threaten the exclusivity of the bond with a reproductive partner (Buss et al., 1992).

\section{Sex Differences in Jealousy}

Multiple studies in a variety of contexts, cultures, and languages have evidenced sex differences in the situations that elicit jealousy in men and women (Buss, 2018; Buunk \& Dijkstra, 2004; Edlund \& Sagarin, 2012). Men are particularly distressed by the loss of sexual exclusivity from their partner, which is directly linked to the adaptive problem of paternity certainty. Women, on the other hand, do not have uncertainty of their maternity, but a major female adaptive challenge is securing commitment and investment from a reproductive partner, consequently emotional infidelity triggers jealousy more intensely than sexual exclusivity in women (Buss, 2013; Trivers, 1972; Symons, 1979). 
Buss et al. (1992) proposed that sex-differences in jealousy can be traced back to Parental Investment Theory (Trivers, 1972), derived from the fact that females in our species have an increased reproductive cost compared to men, and that men who choose to invest in a long-term reproductive partner must secure sexual fidelity to attain fitness (Buss, 2018). Fernandez (2017) specified that female jealousy functions to secure emotional investment, parental involvement, as well as other mate benefits, and to drive away the partner from potential female rivals. So if we consider that adaptations are different according to the restrictions or evolved problems faced by each sex throughout evolution (Symons, 1979), consequently the mating situations that evoke jealousy are different for men and woman. Sex differences in jealousy can be traced back to ancient adaptive problems (Daly, Wilson, \& Weghorst, 1982; Symons, 1979). Specifically, men and women have different adaptive challenges: women have to secure parental investment beyond reproduction, and men have to secure sexual exclusivity to avoid cuckoldry, so that their investment beyond reproduction does not end up in biologically unrelated offspring (Buss, 2018). The empirical evidence has supported that women are more distressed by the possibility of emotional infidelity, which is indicative of resource diversion; and men are more distressed by the possibility of sexual infidelity, which threatens parental investment.

\section{Methodological considerations of the research on Jealousy}

There are over 20 years of prolific research presented to analyze the function of jealousy, which contributed to the description and comprehension of this emotional adaptation (Edlund \& Sagarin, 2012; Bendixen et al, 2015; Buss, 2018). In the original research by Buss et al. (1992), a forced choice method compares the distress produced by two hypothetical scenarios portraying a partner forming a parallel bond with a rival: 1) implying falling in love with the mating rival, or 2) having a sexual relationship with the rival. Sex differences in jealousy resulted in women being more distressed and physiologically reactive to the emotional infidelity more so than the sexual infidelity, while men reacted more against and were more distressed by sexual infidelity than women.

Criticism stated that the forced-choice method to study sex-differences in jealousy had a carryover effect known as "the double-shot hypothesis", which points that women were more distressed by emotional infidelity from men because this also indicates a sexual relationship, while men attributions implied that if their partner were having sex with a rival they must also be in love (see De Steno \& Salovey, 1996; Harris and Christensen, 1996). To falsify the carryover effect, and many other challenges to the biologically adaptive explanation of underlying sex differences in jealousy, different hypothetical scenarios explicitly eliminated the carry over effect (Buss et al., 1999) compared sex-differences in jealousy in multiple cultures, followed up by many intercultural replications (Buss, 2018; Edlund \& Sagarin, 2012).

On another realm, methodological criticism to the forced choice hypothetical scenarios for testing sex differences in jealousy were complemented with the comparison between "sexual or emotional" cues that actual victims of infidelity would remember the most, or the recall of these different cues under cognitive load conditions (Schützwohl, \& Koch, 2004; Schützwohl, 2005), which supported sex differences in jealousy. To illustrate the present state of the art, there is a recent report by Walsh, Millar, \& Westfall, 
(2018), confirming that actual reactions of men and women to real dating infidelity are likely to end the relationship, when the infidelity conforms with sex specific emotional or sexual transgressions for women and men respectively.

\section{Resources allocation benefits in human mating}

Experimental economics developed a method derived from game theory, to represent situations in which all members of a group would benefit from cooperation with the movement of resources, even though there are individual incentives not to cooperate (Kollock, 1998; Hill, 2004). In this context Becker (1976) proposed that different kinds of kinship relationships imply varying degrees of altruism and selfishness from an economic perspective (Becker, 1976). And Evolutionary Psychology identified evidence of kinship and reciprocal social exchange in cooperation between social partners, exemplified in many different contexts (Tooby and Cosmides, 2008).

In this manner, game theory has been a useful model to test cognitive adaptations that underlie actual behavior, leading to decision making rules for facing ancestral problems derived from group living (Cosmides, 1989). As a result of the intersection of these different approaches to human behavior, we know that closeness to a social partner constantly increases cooperation (Freitag and Bauer, 2016; Hackman et al., 2016).

An experimental example of the economic dictator game is when partners respond to a minor anonymity, and the dictators allocate significantly more than when anonymity is complete (Charness and Gneezy, 2008). So if we think of reproductive relationships in terms of social exchange, in this type of dyad individuals can take advantage of all the benefits inherent to a relationship of mutualism and/ or reciprocity (Conroy Beam et al., 2015; Sutcliffe et al., 2011). The maintenance of longterm relationships conveys evolutionary challenges that promote different patterns of commitment, and perceptions about the supply and demand from the partners (Buss y Duntley, 2011). In this context, trust is one of the most well studied emotions, and it has been modeled by game theory, since trust is considered an attribute that responds to long-term social ties (Bhattacharya, Ghosh, Monsivais, Dunbar, \& Kaski, 2017).

\section{Criticism to self-reports and hypothetical jealousy scenarios:}

Predicting emotions or behaviors through self-report has been questioned in terms of ecological validity with real and observable responses (Harris et al., 2002). In addition, this type of measure deploys inferential rather than intuitive reactions (Harmon-Jones et al, 2009). In general, people do not accurately report what they feel or would do in a hypothetical scenario, because it is rather difficult to report a negative and complex emotion in social terms (Lopatosvka and Arapakis, 2011).

One of the principal problems with research on jealousy is that you cannot actually manipulate relationship transgressions without a very high risk of damaging the affective bond of the individuals studied. The challenge then, is to get the emotional response thought to underlie jealousy as close as to the real one, through an experiment with increasing ecological validity.

The use of forced choice hypothetical scenarios, as well as retrospective attempts to recall sexual or emotional infidelity cues have produced much debate regarding the evolutionary hypothesis, since, in many cases, the type of hypothetical scenario and 
forced choice measurement used may carryout confounds (DeSteno, Bartlett, Braverman, \& Salovey, 2002; Harmon-Jones et al. 2009).

\section{The present methodological proposal}

To empirically address adaptive sex differences in jealousy experimentally we sought to find a suitable method to evoke jealousy without resorting to the fictitious situations and/or hypothetical scenarios that have been criticized elsewhere (De Steno, 2010), aiming at improving overall ecological validity of this complex emotion. Experimental evidence for evoking jealousy with the conditions described above are seldom found in the adult literature (for an exception see Buunk and Dijkstra ,2005), but there are a few experiments where there is actual jealousy evocation: Mathes, Adams, \& Davies (1985) examined coping reactions to a rival establishing a romantic relationships via phone calls, De Steno and colleagues (2006) provided evidence of the role of self-esteem in the evocation of jealousy and its link to aggressive behavior, implementing an experiment that evoked jealousy over social encounters in which a member of the couple was rejected in favor of a third party. And finally, Harmon-Jones et al. (2009) developed an empirical tests of the social rejection implied in jealousy, in an experiment where the targets are eyeball interactions generated by computers, and they examine the neural activity associated to social rejection with a modified version of the ostracism paradigm.

Although all these methods have been important advances for experimentally manipulating the precursors of jealousy, we are not aware of direct tests to the function of romantic jealousy to deter away potential rivals, and conversely to secure sex differentiated partner investment (such as the original formulation of Buss et al., 1992 with physiological measures of distress to hypothetical scenarios).

The previous work is of great methodological value for the comprehension of the phenomenon of jealousy, but to our knowledge there are no methods that can be used to test the function of jealousy to protect valued relationships empirically, which will bring about ecological validity.

\section{An economic game for testing sex differences in jealousy}

An evolved strategy that partly solves the problem of mate retention or maintenance of a close cooperating partner is the emotion of jealousy, which is considered a necessary emotion to optimize resources that are at stake in a long-term relationship (Bendixen, Kennair \& Buss, 2015; Conroy Beam et al, 2015). Despite all of this, jealousy has not been addressed directly using economic games, and it is our challenge to do so.

Thus, for the experimental induction of jealousy we sought to modify the classic economic games available, because they are based on a weighting of the self and the other person, but not the interloper. In this case we decided to use a tailored version of the dictator game (Hoffman, McCabe, Shachat, \& Smith, 1994), but without direct incentives for a strictly self-interested dictator (Forsythe,Horowitz, Savin, \& Sefton, 1994). The idea is that the members of the couple play the role of allocator/ receiver in the dictator game with an opposite sex third party, and that the dictator has the opportunity of allocating money to either the partner or the rival. Consequently, the dictator no longer decides whether to distribute between herself and another person, but chooses between a known close person (the partner) and another person of the opposite 
sex. In this way, the benefits of a relationship can be measured in resources, and if these resources are diverted to a rival, we expect that a mate retention strategy should appear (and in this case the emotion induced will be jealousy).

Considering the previous points and knowing that our goal was to achieve model jealousy evoking with changes in the distribution of resources of the classic economic games, we developed a new method to evaluate sex differences in jealousy using an economic game protocol. Specifically we will use a modification of the dictator game that we will call interpersonal dictator game. In this economic game, the first player, "the dictator", determines how to split an endowment (such as a cash prize) between the partner and another person, while the recipient has no influence over the outcome of the game. Sex-differences in jealousy determine that the experimental condition for women has to be similar to emotional infidelity (underlying the adaptive problem of losing parental investment), and that the experimental condition for men must be close to sexual infidelity (which we portrayed as having an interloper investing resources in the partner)

\section{METHODS}

\section{Design}

A mixed ANOVA was used to test the effect of the interpersonal dictator game conditions (within subjects variable) and sex of the participant (between subjects variable) on the reported jealousy after participating on each game.

\section{Participants}

A total of 28 heterosexual couples participated in the experiment $(\mathrm{n}=56)$, with an average age of 24.80 years (range $=23 ; S D=4.7$ ), and a mean relationship duration of 39 months (range $=162 ; S D=41.2$ ). Each dyad came to the laboratory, where they separately but simultaneously participated in the interpersonal dictator game in exchange for an approximately \$7.5 USD incentive, implemented through the Qualtrics TM platform.

\section{Procedure}

The experiment followed the same order for all participants: Signing the informed consent, completing a demographic questionnaire, and then each treatment condition (three interpersonal dictator game scenarios), followed by a subjective assessment about how much jealousy the game evoked. Couples played at the same time and they believed they were playing online with a third party first, and then with their partner.

\section{Interpersonal dictator game}

We presented two counterbalanced dictator games followed by a control condition. Each condition is a modified version of the economic game, in which the dictator allocates or receives money from the partner or an opposite sex person. The rationale is that the 
members of the dyad perform both: the role of allocator and the role of recipient. The outcome of each game is immediately informed to the partner.

The experimental condition aimed at representing sex specific jealousy evoking conditions in hunter-gatherers contexts where jealousy would play a retentive role: 1) for women, the game represents a situation in which her male partner allocates resources to another female (emotional infidelity), and 2) for men represents a situation in which his female partner receives resources from another male (sexual infidelity). The control condition 1) for women represents their male partner receiving money from another woman; and 2) for men, it represents their female partner giving money to another man. The neutral condition does not involve allocating/receiving resources from the partner, but rather portraits 1) "any man" (for the female participants), or 2) "any woman" (for the male participants) allocating or receiving money to/from another man. Each participant experienced the three scenarios and after each condition the players indicated how jealous the situation would make them (see Figure 1 for an illustration of the jealousy evoking protocol, with the conditions depicted by sex).

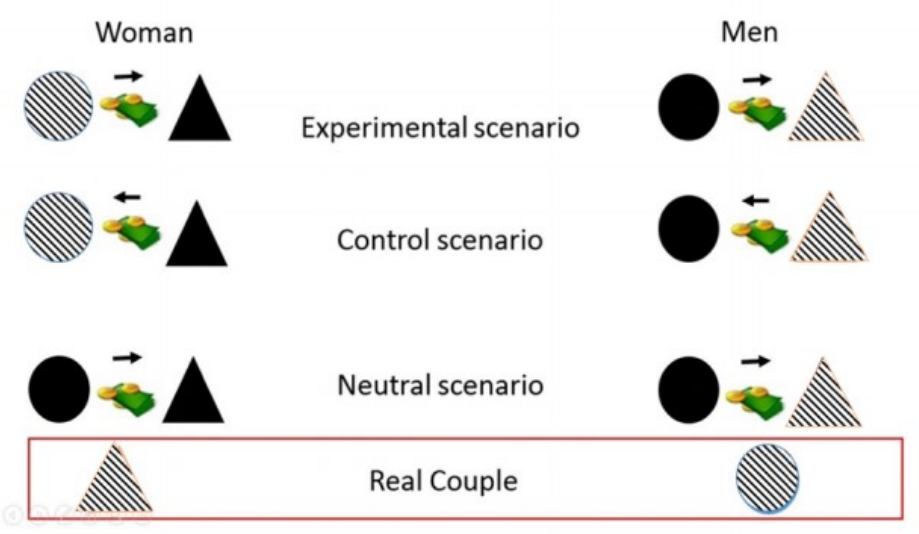

Figure 1: Interpersonal dictator game.

The red box shows the real couple. The triangle is the woman and the circle is the man. Another "woman" as recipient and allocator is the manipulation for the evocation of jealousy for the female member of the dyad, while for the male partner the manipulation is another "man" giving to, or receiving money from their partner. In black are the individuals who play as rivals for the distribution of money.

\section{RESULTS AND DISCUSSION}

The results from the mixed ANOVA yielded a significantly effect of interpersonal dictator game condition on jealousy $\left(F_{2,54}=21.02 ; p<.001 ; \eta^{2}=0.28 ; 1-p=1 ; S S=\right.$ $42.04)$, but there was no significant interaction by $\operatorname{sex}\left(F_{1,54}=1.95 ; p=0.16\right)$.

The lack of interaction of the conditions and sex could be explained due to the low sample size that increases Type II error, but it could also be explained by the economic 
game design: since the experimental and control condition are tailored to sex-specific jealousy evoking conditions (allocating to/receiving from) towards the rival, so no interaction should emerge.

For example, in the case of women, an emotional infidelity is marked by the diversion of commitment and resource allocation in the relationship, and the economic game seems to conform in similar ways with this evolutionary assumption, because it explicitly expresses that the partner distributes resources to another woman. In contrast, the evolutionary function of men jealousy is to avoid sexual infidelity from his long-term partner (Buss, 2015), but the target experimental condition for men was aimed at evoking this situation when another third party offers resources to his partner.

Secondly, to determine if jealousy was differentially evoked by the specific interpersonal game conditions, A post-hoc Tukey test showed all conditions where significantly different $(p=<.05$; see Figure 2$)$. This result is very important, because it adds evidence to evolved sex differences in jealousy using an interpersonal dictator game, since the distribution of resources with a third party resulted relevant for the evocation of jealousy.

On the other hand, there is a significant main effect of $\operatorname{sex}\left(F_{1,54}=6.93 ; p<.001\right)$, suggesting that the experimental condition (testing the evolutionary hypothesis) evokes more jealousy than the control and neutral condition in women compared to men (see Figure 2).

The results of this pilot method allow for continuing testing the target interpersonal dictator game as an effective protocol to activate more reproductive jealousy in the sexspecific resource distribution experimental condition, compared to a control and a neutral condition, differentiated by sex.

It should be added that an informal registry of the thoughts and impressions after participation in the game was marked by some women expressing discomfort, nervousness and tension with the results of the conditions of the game before debriefing. Literally one of them expressed: "the game put me in a tense situation". This allowed us to conclude that the interpersonal dictator game may allow for making observations about the real behavior in a context of cooperation with the partner without having to elaborate an artificial scenario. Future directions to complement these initial findings will be to look at the effect of the dictator game conditions on other emotions. Since jealousy is considered a complex emotion that evolved to guard the loss of a valued relationship to a rival (Buss, 2018; Bunk, 1997), which could evoke basic emotions or a mixture of these (Sabini and Green, 2004; Pietrzak et al, 2002; Guerrero, Trost and Yoshimura, 2005; Shackelford et al, 2000). Future extensions of this research should extend the analysis of other basic and complex emotions after exposing the results of each game (such as happiness, disgust, sadness, anger).

In sum, we were able to pilot and measure the actual behavioral induction of jealousy through an interpersonal dictator game tailored for women and men. Although this report shows an initial approach for experimentally manipulating the conditions that have been repeatedly found to induce jealousy in past research, we do find that in real heterosexual couples the effect of a partner diverting resources to another woman and the allocation of resources from another man to the partner, as efficient triggers for jealousy in women and men (respectively). One important limitation, in addition to the pilot nature and small sample reported here, is that there is a tendency for the 
experimental game to better evoke the evolved adaptive problem for women than for men, because we are only manipulating the jealousy evoking effect of resource allocation from another man to the partner in men, but this is not a complete equivalent of sexual infidelity from a woman. We aim to find a better treatment to similarly induce jealousy in men as well as in women with a sex-specific economic game situation that may be similar to sexual infidelity.

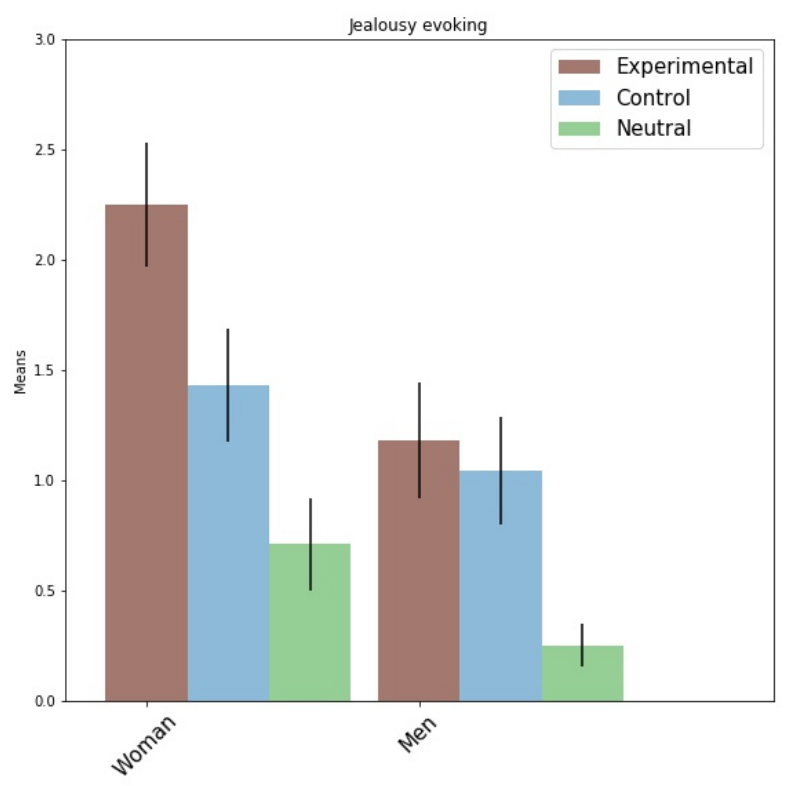

Figure 2: Jealousy evoking protocol: for woman and men. We observed three conditions where the means are significantly different from each other as determined by a Tukey's test.

\section{ACKNOWLEDGMENTS}

This research was supported by Fondo Nacional de Desarrollo Científico y Tecnológico of the Government of Chile: Fondecyt Grant \#1181114. 


\section{REFERENCES}

Bendixen, M., Kennair, L. E. O., \& Buss, D. M. (2015). Jealousy: Evidence of strong sex differences using both forced choice and continuous measure paradigms. Personality and Individual Differences, 86, 212-216. DOI

Becker Gary S. (1976). Altruism, Egoism, and Genetic Fitness: Economics and Sociobiology. Journal of Economic Literature, 14(3), 817-826.

Bhattacharya, K., Ghosh, A., Monsivais, D., Dunbar, R., \& Kaski, K. (2017). Absence makes the heart grow fonder: social compensation when failure to interact risks weakening a relationship. EPJ Data Science, 6(1). DOI

Buss, D. M. (1989). Conflict between the sexes: Strategic interference and the evocation of anger and upset. Journal of Personality and Social Psychology, 56(5), 735-747. DOI

Buss, D. M., Larsen, R. J., Westen, D., \& Semmelroth, J. (1992). Sex Differences in Jealousy: Evolution, Physiology, and Psychology. Psychological Science, 3(4), 251-256. DOI

Buss D. M., Shackelford, T. K., Kirkpatrick, L. A., Choe, J. C., Lim, H. K., Hasegawa, et al. (1999). Jealousy and the nature of beliefs about infidelity: Tests of competing hypotheses about sex differences in the United States, Korea, and Japan. Personal Relationships, 6(1), 125-150. DOI

Buss, D. M. (2015). Mating. The Handbook of Evolutionary Psychology, 251-257. DOI

Buss, D. M., \& Haselton, M. (2005a). The evolution of jealousy. Trends in Cognitive Sciences, 9(11), 506-507. DOI

Buss, D. M. (2005b). Sex differences in the design features of socially contingent mating adaptations. Behavioral and Brain Sciences, 28(02). DOI

Buss, D. M., \& Duntley, J. D. (2011). The evolution of intimate partner violence. Aggression and Violent Behavior, 16(5), 411-419. DOI

Buss, D. M. (2018). Sexual and Emotional Infidelity: Evolved Gender Differences in Jealousy Prove Robust and Replicable. Perspectives on Psychological Science, 13(2), 155-160. DOI

Burkett, B. N., (2009). Friendship, jealousy, and the Banker's Paradox. Unpublished doctoral dissertation. University of California, Santa Barbara, California.

Buunk, B. P. (1997). Personality, birth order and attachment styles as related to various types of jealousy. Personality and Individual Differences, 23(6), 997-1006. DOI

Buunk, B. P., \& Dijkstra, P. (2005). A narrow waist versus broad shoulders: Sex and age differences in the jealousy-evoking characteristics of a rival's body build. Personality and Individual Differences, 39(2), 379-389. DOI

Charness, G., Gneezy, U. (2008). What's in a name? Anonymity and social distance in dictator and ultimatum games. Journal of Economic Behavior \& Organization, 68, 29-35. DOI

Chin, K., Atkinson, B. E., Raheb, H., Harris, E., \& Vernon, P. A. (2017). The dark side of romantic jealousy. Personality and Individual Differences, 115, 23-29. DOI

Conroy-Beam, D., Goetz, C. D., \& Buss, D. M. (2015). Why Do Humans Form Long-Term Mateships? An Evolutionary Game-Theoretic Model. Advances in Experimental Social Psychology, 1-39. DOI

Cosmides, L. (1989). The logic of social exchange: Has natural selection shaped how humans reason? Studies with the Wason selection task. Cognition, 31(3), 187-276. DOI

Cosmides, L., \& Tooby, J. (2013). Evolutionary Psychology: New Perspectives on Cognition and Motivation. Annual Review of Psychology, 64(1), 201-229. DOI

Daly, M., Wilson, M., \& Weghorst, S. J. (1982). Male sexual jealousy. Ethology and Sociobiology, $3(1), 11-27$. DOI 
DeSteno, D., Bartlett, M. Y., Braverman, J., \& Salovey, P. (2002). Sex differences in jealousy: Evolutionary mechanism or artifact of measurement? Journal of Personality and Social Psychology, 83(5), 1103-1116. DOI

DeSteno, D., Valdesolo, P., \& Bartlett, M. Y. (2006). Jealousy and the threatened self: Getting to the heart of the green-eyed monster. Journal of Personality and Social Psychology, 91(4), 626-641. DOI

DeSteno, D. (2010). Mismeasuring Jealousy. Psychological Science, 21(9), 1355-1356. DOI

Dillon, L. (2013). Functional aspects of jealousy across the lifespan. Human Ethology Bulletin 28(2), 13-26.

Dijkstra, P., \& Buunk, B. P. (2002). Sex differences in the jealousy-evoking effect of rival characteristics. European Journal of Social Psychology, 32(6), 829-852. DOI

Foster, J. D., Jonason, P. K., Shrira, I., Keith Campbell, W., Shiverdecker, L. K., \& Varner, S. C. (2014). What do you get when you make somebody else's partner your own? An analysis of relationships formed via mate poaching. Journal of Research in Personality, 52, 78-90. DOI

Forsythe, R., Horowitz, J. L., Savin, N. E., \& Sefton, M. (1994). Fairness in Simple Bargaining Experiments. Games and Economic Behavior, 6(3), 347-369. DOI

Freitag, M., \& Bauer, P. C. (2016). Personality traits and the propensity to trust friends and strangers. The Social Science Journal, 53(4), 467-476. DOI

Guerrero, L. K., Trost, M. R., \& Yoshimura, S. M. (2005). Romantic jealousy: Emotions and communicative responses. Personal Relationships, 12(2), 233-252. DOI

Harmon-Jones, E., Peterson, C. K., \& Harris, C. R. (2009). Jealousy: Novel methods and neural correlates. Emotion, 9(1), 113-117. DOI

Hackman, J., Munira, S., Jasmin, K., \& Hruschka, D. (2016). Revisiting Psychological Mechanisms in the Anthropology of Altruism. Human Nature, 28(1), 76-91. DOI

Hart, S. L. (2018). Jealousy and attachment: Adaptations to threat posed by the birth of a sibling. Evolutionary Behavioral Sciences, 12(4), 263-275. DOI

Harris, C. R. (2002). Sexual and Romantic Jealousy in Heterosexual and Homosexual Adults. Psychological Science, 13(1), 7-12. DOI

Hill, S. E., \& Reeve, H. K. (2004). Mating games: the evolution of human mating transactions. Behavioral Ecology, 15(5), 748-756. DOI

Hoffman, E., McCabe, K., Shachat, K., \& Smith, V. (1994). Preferences, Property Rights, and Anonymity in Bargaining Games. Games and Economic Behavior, 7(3), 346-380. DOI

Ellis, M., \& Kleinplatz, P. J. (2018). How contingencies of self-worth influence reactions to emotional and sexual infidelity. The Canadian Journal of Human Sexuality, 27(1), 43-54. DOI

Kollock, P. (1998). Social Dilemmas: The Anatomy of Cooperation. Annual Review of Sociology, 24(1), 183-214. DOI

Lopatovska, I., \& Arapakis, I. (2011). Theories, methods and current research on emotions in library and information science, information retrieval and human-computer interaction. Information Processing \& Management, 47(4), 575-592. DOI

Mathes, E. W., Adams, H. E., \& Davies, R. M. (1985). Jealousy: Loss of relationship rewards, loss of self-esteem, depression, anxiety, and anger. Journal of Personality and Social Psychology, $48(6), 1552-1561$. DOI

Pietrzak, R. (2002). Sex differences in human jealousy A coordinated study of forced-choice, continuous rating-scale, and physiological responses on the same subjects. Evolution and Human Behavior, 23(2), 83-94. DOI 
Sabini, J., \& Green, M. C. (2004). Emotional Responses to Sexual and Emotional Infidelity: Constants and Differences Across Genders, Samples, and Methods. Personality and Social Psychology Bulletin, 30(11), 1375-1388. DOI

Sagarin, B. J., Martin, A. L., Coutinho, S. A., Edlund, J. E., Patel, L., Skowronski, J. J., \& Zengel, B. (2012). Sex differences in jealousy: a meta-analytic examination. Evolution and Human Behavior, 33(6), 595-614. DOI

Sutcliffe, A., Dunbar, R., Binder, J., \& Arrow, H. (2011). Relationships and the social brain: Integrating psychological and evolutionary perspectives. British Journal of Psychology, 103(2), 149-168. DOI

Symons, D. (1980). The evolution of human sexuality revisited. Behavioral and Brain Sciences, 3(02), 203. DOI

Shackelford, T. K., LeBlanc, G. J., \& Drass, E. (2000). Emotional reactions to infidelity. Cognition \& Emotion, 14(5), 643-659. DOI

Schmitt, D. P., \& Buss, D. M. (2001). Human mate poaching: Tactics and temptations for infiltrating existing mateships. Journal of Personality and Social Psychology, 80(6), 894917. DOI

Schützwohl, A., \& Koch, S. (2004). Sex differences in jealousy: The recall of cues to sexual and emotional infidelity in personally more and less threatening context conditions. Evolution and Human Behavior, 25(4), 249-257. DOI

Schützwohl, A. (2005). Sex differences in jealousy: the processing of cues to infidelity. Evolution and Human Behavior, 26(3), 288-299. DOI

Tooby, J. \& Cosmides, L. (2008). The evolutionary psychology of the emotions and their relationship to internal regulatory variables. In M. Lewis, J. M. Haviland-Jones \& L. F. Barrett, Handbook of Emotions, 3, 114-137

Trivers, R. L. (Ed.). (2017). Parental Investment and Sexual Selection. Sexual Selection and the Descent of Man, 136-179. DOI

Walsh, M., Millar, M., \& Westfall, R. S. (2018). Sex Differences in Responses to Emotional and Sexual Infidelity in Dating Relationships. Journal of Individual Differences, 1-8. DOI 